\section{BRAZIULIAN JOURNAL}

OF MEDICAL AND BIOLOGICAL RESHARCH

www.bjournal.com.br
ISSN 0100-879X

Volume 43 (11) 1010-1134 November 2010

BIOMEDICAL SCIENCES

AND

CLINICAL INVESTIGATION

Braz J Med Biol Res, November 2010, Volume 43(11) 1054-1061

doi: 10.1590/S0100-879X2010007500108

In vitro activity of hypnophilin from Lentinus strigosus: a potential prototype for Chagas disease and leishmaniasis chemotherapy

E.M. Souza-Fagundes, B.B. Cota, L.H. Rosa, A.J. Romanha, R. Corrêa-Oliveira, C.A. Rosa, C.L. Zani, A. Teixeira-Carvalho and O.A. Martins-Filho

The Brazilian Journal of Medical and Biological Research is partially financed by
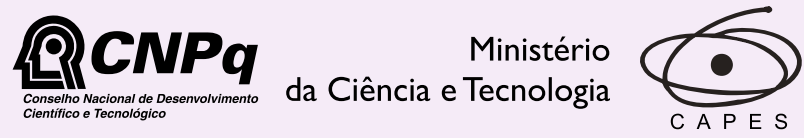

Ministério da Educação

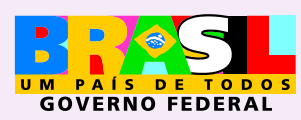

TFAPESP

Institutional Sponsors
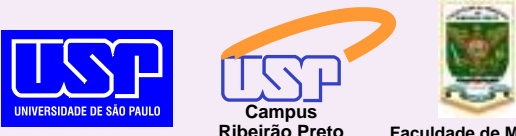

$\oplus$ SHIMADZU

GE Healthcare

Hotsite of proteomics metabolomics developped by:

\title{
现
}




\title{
In vitro activity of hypnophilin from Lentinus strigosus: a potential prototype for Chagas disease and leishmaniasis chemotherapy
}

\author{
E.M. Souza-Fagundes ${ }^{1,6}$, B.B. Cota ${ }^{3}$, L.H. Rosa ${ }^{7}$, A.J. Romanha ${ }^{4}$, R. Corrêa-Oliveira ${ }^{5}$, \\ C.A. Rosa ${ }^{2}$, C.L. Zani ${ }^{3}$, A. Teixeira-Carvalho ${ }^{6}$ and O.A. Martins-Filho 6 \\ ${ }^{1}$ Departamento de Fisiologia e Biofísica, ${ }^{2}$ Departamento de Microbiologia, Instituto de Ciências Biológicas, \\ Universidade Federal de Minas Gerais, Belo Horizonte, MG, Brasil \\ ${ }^{3}$ Laboratório de Química de Produtos Naturais, ${ }^{4}$ Laboratório de Parasitologia Celular e Molecular, \\ ${ }^{5}$ Laboratório de Imunologia Celular e Molecular, ${ }^{6}$ Laboratório de Biomarcadores de Diagnóstico e Monitoração, \\ Centro de Pesquisas René Rachou, FIOCRUZ-MG, Belo Horizonte, MG, Brasil \\ ${ }^{7}$ Laboratório de Microbiologia, Instituto de Ciências Exatas e Biológicas, Universidade Federal de Ouro Preto, \\ Ouro Preto, MG, Brasil
}

\begin{abstract}
Hypnophilin and panepoxydone, terpenoids isolated from Lentinus strigosus, have significant inhibitory activity on Trypanosoma cruzi trypanothione reductase (TR). Although they have similar TR inhibitory activity at $10 \mu \mathrm{g} / \mathrm{mL}(40.3 \mu \mathrm{M}$ and 47.6 $\mu \mathrm{M}$ for hypnophilin and panepoxydone, respectively; $\sim 100 \%)$, hypnophilin has a slightly greater inhibitory activity ( $71 \%)$ on T. cruzi amastigote (AMA) growth in vitro as well as on in vitro phytohemagglutinin (PHA)-induced peripheral blood mononuclear (PBMC) proliferation ( 70\%) compared to panepoxydone (69\% AMA inhibition and 91\% PBMC inhibition). Hypnophilin and panepoxydone at $1.25 \mu \mathrm{g} / \mathrm{mL}$ had $67 \%$ inhibitory activity on Leishmania (Leishmania) amazonensis amastigote-like (AMA-like) growth in vitro. The panepoxydone activity was accompanied by a significant inhibitory effect on PHA-induced PBMC proliferation, suggesting a cytotoxic action. Moreover, incubation of human PBMC with panepoxydone reduced the percentage of $\mathrm{CD} 16^{+}$and $\mathrm{CD} 14^{+}$cells and down-regulated $\mathrm{CD} 19^{+}, \mathrm{CD} 4^{+}$and $\mathrm{CD} 8^{+}$cells, while hypnophilin did not alter any of the phenotypes analyzed. These data indicate that hypnophilin may be considered to be a prototype for the design of drugs for the chemotherapy of diseases caused by Trypanosomatidae.
\end{abstract}

Key words: Chagas disease; Leishmaniasis; Chemotherapy; Immunomodulation; Hypnophilin; Lentinus strigosus

\section{Introduction}

Programs to promote the development of pharmaceutical products for the treatment of so-called neglected diseases have been implemented around the world (1). Neglected diseases include diseases that disproportionately affect millions of poor populations in developing countries, such as tuberculosis, malaria, leishmaniasis, and trypanosomiasis. There is an urgent need for novel and improved drugs against these tropical diseases, specifically those caused by protozoa (2). Trypanosomatidae alone are responsible for an infected population of nearly 30 million, and more than 400 million are at risk depending on medical care and on new perspectives of chemotherapy (3).

The drugs currently used for the treatment of Chagas disease are two nitroaromatic heterocyles, nifurtimo ${ }^{\circledR}[\mathrm{Nfx}$, Lampit Bayer, which is produced and used predominantly in Central America (4)] and benznidazole (Bnz, Rochagan ${ }^{\circledR}$, Roche, Switzerland) that display little or no activity during chronic infection, despite their beneficial effects during acute disease $(3,5,6)$. The drugs of choice for the treatment of leishmaniasis are sodium stibogluconate (Pentostam ${ }^{\circledR}$, GlaxoSmithKline, UK), meglumine antimoniate (Glucantime $^{\circledR}$, Sanofi-Aventis, France), pentamidine (Astellas

Correspondence: O.A. Martins-Filho, Laboratório de Biomarcadores de Diagnóstico e Monitoração, Centro de Pesquisas René Rachou, FIOCRUZ-MG, Av. Augusto de Lima, 1715, 30190-002 Belo Horizonte, MG, Brasil. Fax: +55-31-3295-3115.

E-mail: andreat@cpqrr.fiocruz.br

Received December 3, 2009. Accepted September 15, 2010. Available online October 15, 2010. Published November $12,2010$. 
Healthcare Inc., USA) and liposomal amphotericin $B^{\circledR}$ (Bristol-Myers Squibb, USA) but they sometimes fail to act (3). Furthermore, these drugs have several side effects that reduce their clinical use $(4,7)$.

Trypanothione reductase (TR) is an essential enzyme specific for the parasitic protozoa of the order Kinetoplastida. The enzyme catalyzes the NADPH-dependent reduction of glutathionylspermidine conjugates. It is a key enzyme of the parasite's thiol metabolism in which the ubiquitous glutathione/glutathione reductase system is replaced by trypanothione and TR. Reduction of trypanothione disulfide is crucial for the maintenance of a reducing intracellular milieu. These considerations render the parasite-specific TR an attractive target molecule for the development of structure-based drug against Chagas disease, African sleeping sickness, other diseases caused by trypanosomes, and leishmanias $(8,9)$. Studies have also been devoted to the characterization of the impact of new drug prototypes on the human immune system (10).

We initially screened more than 3000 extracts of plants and fungi and observed that the extract of Lentinus strigosus, a Basidiomycete fungus belonging to the Polyporaceae family $(11,12)$, contained the most promising drug activity because of its strong TR-inhibitory activity. This TR-bioguided assay led to a detailed investigation of two secondary metabolites produced by the fungus $L$. strigosus named panepoxydone and hypnophilin (12)

In the present study, we provide evidence for the differential cytotoxic effect of these drugs on the proliferation of human peripheral blood mononuclear cells (PBMC) stimulated by phytohemagglutinin (PHA) and on the immunophenotyping of normal PBMC. These compounds also had a significant antiparasitic effect against Trypanosoma cruzi amastigotes (AMA) and AMA-like forms of Leishmania (Leishmania) amazonensis. We also report for the first time the effect of hypnophilin and panepoxydone on leukocyte subpopulations, demonstrating a differential cytotoxic effect on lymphocytes. Our findings suggest that hypnophilin, a terpenoid from L. strigosus, is a potential prototype for Chagas disease and leishmaniasis chemotherapy.

\section{Material and Methods}

\section{Panepoxydone and hypnophilin}

Panepoxydone and hypnophilin were isolated from an ethyl acetate extract of $L$. strigosus (Schwein) Fr. (voucher code UFMGCB975) as previously described (12). All spectral data (MS, IR, 1H-, and 13C-NMR) of the crystals obtained for this study were in agreement with that published for panepoxydone and hypnophilin (12) (Figure 1). The compounds were dissolved in dimethylsulfoxide (DMSO), diluted in RPMI and added to the culture at the desired final concentrations. A control experiment was performed using $0.05 \%$ DMSO, the maximum concentration of DMSO used in the present study.

\section{Measurement of recombinant $T$. cruzi trypanothione reductase}

The colorimetric assay was performed in 96-well plates (Costar 9017, Corning, USA) by the method of Hamilton et al. (13) using DTNB (17.5 nmol) as the colorimetric reagent. Each assay well $(250 \mu \mathrm{L})$ contained enzyme (6 $\mathrm{mU})$, trypanothione $(0.25 \mathrm{nmol})$ and NADPH (50 nmol) using HEPES buffer ( $40 \mathrm{mM}$, pH 7.5) with $1 \mathrm{mM}$ EDTA. The experiments were conducted in triplicate and the results are reported as percent enzyme inhibition. The control drug was clomipramine (6.5 $\mu \mathrm{M}$, Sigma, USA), a known TR inhibitor (14). The assays were conducted after 30 min at $30^{\circ} \mathrm{C}$ of pre-incubation of the compounds with the enzyme before the addition of the NADPH co-factor and the substrate trypanothione. Measurements were made at $412 \mathrm{~nm}$ in the kinetic mode every $10 \mathrm{~s}$ for $5 \mathrm{~min}$ in triplicate in two independent experiments.

\section{Measurement of $T$. cruzi amastigote $\beta$-galactosidase activity}

The assay was performed by the method of Buckner et al. (15). T. cruzi (Tulahuen strain) expressing the Escherichia coli $\beta$-galactosidase gene was grown on a monolayer of mouse L929 fibroblasts. Cultures to be assayed for $\beta$-galactosidase activity were grown in RPMI 1640 medium, $\mathrm{pH}$ 7.2-7.4, without phenol red (Gibco BRL, USA) plus $10 \%$ fetal bovine serum, $2 \mathrm{mM}$ glutamine and $40 \mu \mathrm{g} / \mathrm{mL}$ gentamicin. For the bioassay, 4000 L929 cells in $80 \mu \mathrm{L}$ supplemented medium were added to each well of a 96-well microtiter plate. After incubation overnight, 40,000 trypomastigotes in $20 \mu \mathrm{L}$ were added to the cells and incubated for $2 \mathrm{~h}$. The medium containing extracellular parasites was replaced with $200 \mu \mathrm{L}$ of fresh medium and the plate was incubated for an additional $48 \mathrm{~h}$ to establish the infection. The medium was then replaced with solutions of the compounds at different concentrations in fresh medium $(200 \mu \mathrm{L})$, and the plate was incubated for $96 \mathrm{~h}$. Then, $50 \mu \mathrm{L}$ $500 \mu \mathrm{M}$ chlorophenol red glycoside in $0.5 \%$ Nonidet P40 was added to each well, the plate was incubated for $24 \mathrm{~h}$, and absorbance at $570 \mathrm{~nm}$ was measured. Controls with uninfected cells, untreated infected cells, and medium only were run in parallel. The results are reported as percent reduction of absorbance at $570 \mathrm{~nm}$ in the experimental wells compared to control wells with untreated infected cells (percent inhibition $\left.=\left(1-\mathrm{Abs}_{\mathrm{exp}} / \mathrm{Abs}_{\mathrm{contr}}\right) \times 100\right)$. Triplicate analyses were run in the same plate, and the experiments were repeated at least once.

\section{Percent inhibition of the viability of amastigote-like forms of $L$. (L.) amazonensis by hypnophilin and panepoxydone}

Promastigotes of L. (L.) amazonensis (IFLA/BR/196/ $\mathrm{PH}-8$ strain) were obtained from lesions of infected hamsters. The parasites were grown at $26^{\circ} \mathrm{C}$ in Schneider's

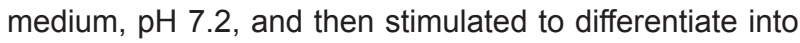


the amastigote forms by increasing the temperature $\left(32^{\circ} \mathrm{C}\right)$ and lowering the $\mathrm{pH}$ of the Schneider's medium to 6.0. After 7 days under these conditions, $90 \%$ of the promastigotes had transformed into amastigote-like forms, which were then used in the bioassays. Amastigote density was adjusted to $1 \times 10^{8}$ parasites $/ \mathrm{mL}$, and $90 \mu \mathrm{L}$ added to each well of 96 well plates. Ten microliters of the test and control solutions was added to attain the desired concentrations. The plates were incubated at $32^{\circ} \mathrm{C}$ for $72 \mathrm{~h}$, and cell viability was then determined using the methyl thiazolyl tetrazolium (MTT) assay (16). The results are reported as percent inhibition in relation to the controls without the drug. Amphotericin $B$ at $0.2 \mu \mathrm{g} / \mathrm{mL}$ (Fungisons Bristol-Myers Squibb B, Brazil) was used as a positive control. All assays were performed in triplicate.

\section{Percent inhibition of human PBMC proliferation by hypnophilin and panepoxydone}

Hypnophilin and panepoxydone at $10 \mu \mathrm{g} / \mathrm{mL}$ were used to determine their effect on in vitro PHA-induced PBMC proliferation. PBMC were obtained from healthy adult volunteers and processed as previously described (17). The assay was performed by the method of Souza-Fagundes et al. (10). Briefly, the cultures were incubated with samples in the presence or absence of dexamethasone (positive control) and cell proliferation and viability were determined by a colorimetric assay using MTT (16). The results are reported as percent inhibition or stimulation compared to PHA-induced lymphocyte proliferation without added drugs.

\section{Immunophenotyping of PBMC}

PBMC ( $2 \times 10^{6}$ cells $\left./ \mathrm{mL}\right)$ were cultured in polypropylene tubes (Falcon, 2063, Becton Dickinson, USA) in the presence of $1.25 \mu \mathrm{g} / \mathrm{mL}$ panepoxydone or hypnophilin for $18 \mathrm{~h}$. Immunophenotyping was performed using $2 \mu \mathrm{L}$ of the following antibody clones (Becton Dickinson): Leu-4 FITC (CD3), Leu-3a FITC (CD4), Leu-2a FITC (CD8), and Leu-M3 PE (CD14). The isotype controls used were phycoerythrin-conjugated IgG1 and FITC-conjugated IgG2. Data acquisition and analysis were performed with a single-laser flow cytometer (FACscan, Becton Dickinson) using the CellQuest software. Ten thousand events were counted for each determination. Laser scatter dot plots (size versus granularity) were used to identify and select the PBMC subpopulations. Two-dimensional fluorescence dot plots (FL1 versus FL2) were used to identify labeled cell subpopulations after staining with fluorochrome-conjugated monoclonal antibodies.

All experimental protocols were approved by the Ethics Committee of Fundação Oswaldo Cruz, Ministry of Health, Brazil.

\section{Statistical analysis}

Each experiment with PBMC and purified cells was run in triplicate and repeated at least five times on different days using PBMC from different individuals. Data are reported as means \pm SEM. All data for each experiment were analyzed by the Student $t$-test. Statistical significance was considered when $P$ was $\leq 0.05$. The 50 and $90 \%$ effective concentrations $\left(E_{50}\right.$ and $\mathrm{EC}_{90}$, respectively) of panepoxydone and hypnophilin against parasite growth were determined by a sigmoidal Hill function with the GraphPad Prism software (version 5; GraphPad Software Inc., USA) and linear regression analysis with the MS EXCEL ${ }^{\circledR}$ version 2007 (Microsoft Inc., USA).

\section{Results}

Inhibition of in vitro TR activity, T. cruzi amastigote growth, L. (L.) amazonensis growth and PHA-induced human PBMC proliferation by panepoxydone and hypnophilin

The chemical structures of panepoxydone and hypnophilin isolated from the organic extract of $L$. strigosus are shown in Figure 1. We evaluated their inhibitory effect in vitro in order to mimic the in vivo situation of parasite growth. Human PBMC proliferation assays were performed to investigate the impact of new drug prototypes on the human immune system (Figure 1). Both compounds at $10 \mu \mathrm{g} /$ $\mathrm{mL}$ (47.6 and $40.3 \mu \mathrm{M}$ for panepoxydone and hypnophilin, respectively) significantly inhibited TR activity, growth of $T$. cruzi or $L$. (L.) amazonensis amastigotes, as well as the PHA-induced proliferation of human PBMC.

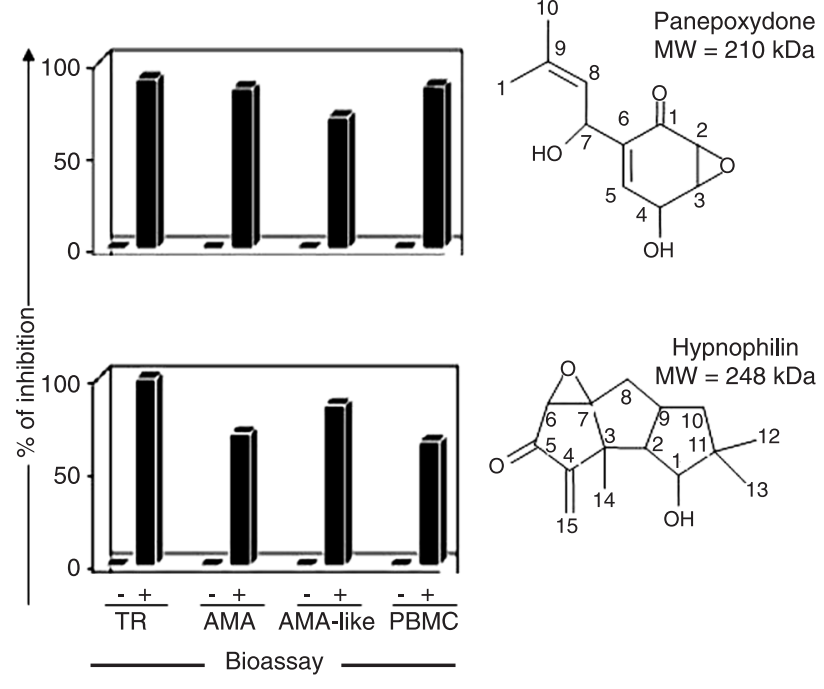

Figure 1. Effect of panepoxydone and hypnophilin on trypanothione reductase (TR) activity, on the viability of amastigotes of Trypanosoma cruzi (AMA) and of amastigote-like forms of Leishmania (Leishmania) amazonensis (AMA-like), and on phytohemagglutinin-induced human peripheral blood mononuclear cell (PBMC) proliferation. The concentration of each compound added to the cultures (+) was 10 $\mu \mathrm{g} / \mathrm{mL}$. Untreated cultures are shown as (-). Data are reported as means. The structures of the compounds are shown on the right. 


\section{Dose-response relationships}

The data in Figure 2A show that panepoxydone and hypnophilin both present a typical dose-response inhibition of TR, $T$. cruzi AMA, amastigotes of $L$. (L.) amazonensis
(AMA-like), and PHA-induced human PBMC lymphoproliferation.

As shown in Table 1, the EC values for hypnophilin demonstrated a higher inhibitory effect on T. cruzi AMA

A
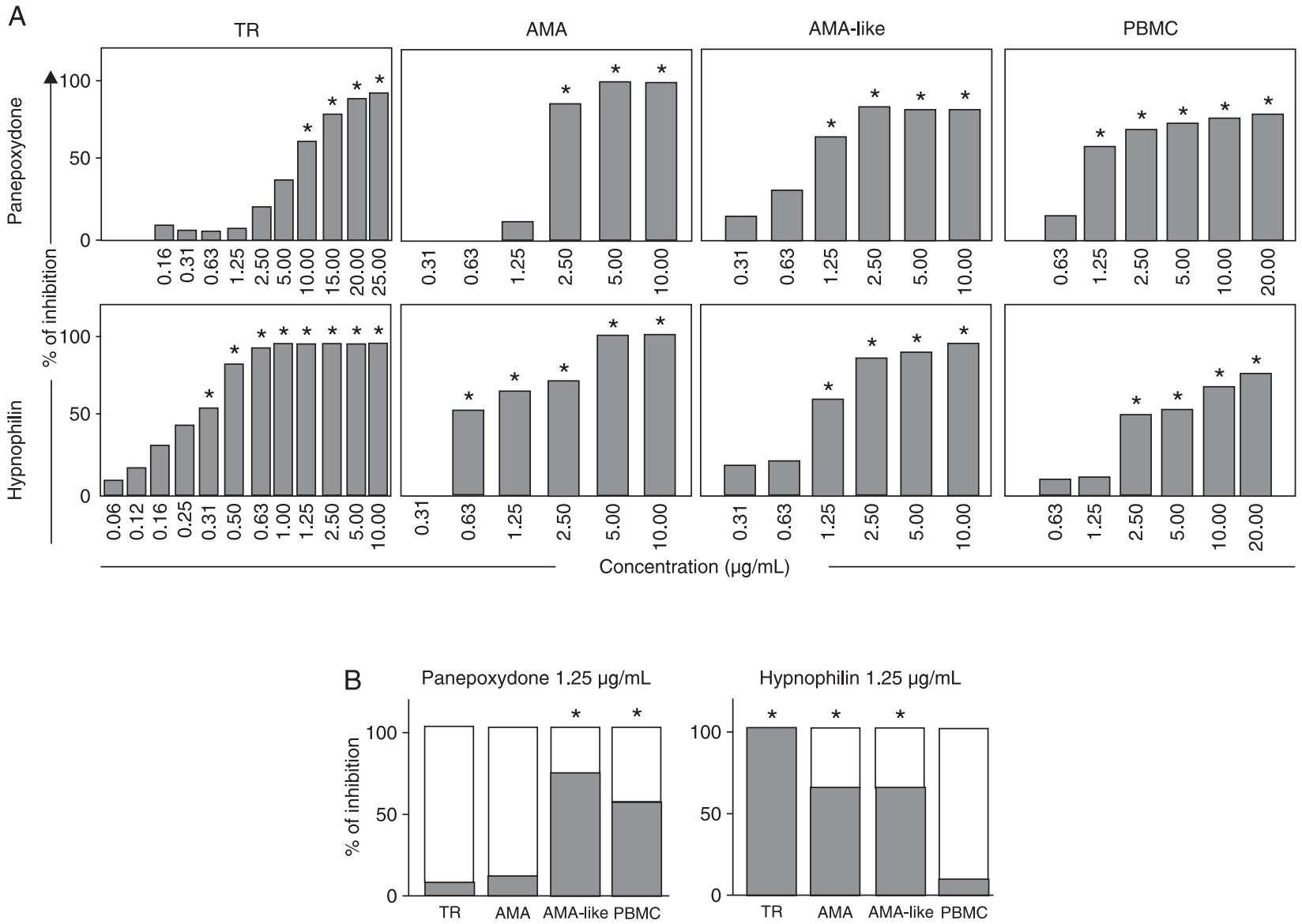

Figure 2. Dose-response effect of panepoxydone and hypnophilin on trypanothione reductase (TR), amastigotes of Trypanosoma cruzi (AMA), amastigote-like forms of Leishmania (Leishmania) amazonensis (AMA-like) and phytohemagglutinin-induced human lymphoproliferation (PBMC) assays (A). The concentration of $1.25 \mu \mathrm{g} / \mathrm{mL}$ was considered to guarantee higher AMA-like inhibitory capacity with a well-preserved PBMC response in the presence of the substances $(B)$. The statistically significant differences $(P<0.05)$ were obtained by the paired Student $t$-test and are indicated by asterisks.

Table 1. In vitro activity $(\mu \mathrm{g} / \mathrm{mL}$ or $\mu \mathrm{M})$ of panepoxydone and hypnophilin against Trypanosoma cruzi amastigotes (AMA) and Leishmania (Leishmania) amazonensis amastigotes (AMA-like).

\begin{tabular}{lccccr}
\hline In vitro assays & \multicolumn{2}{c}{ Hypnophilin $(\mu \mathrm{g} / \mathrm{mL})$} & & \multicolumn{2}{c}{ Panepoxydone $(\mu \mathrm{g} / \mathrm{mL})$} \\
\cline { 2 - 3 } \cline { 5 - 6 } & EC $_{50}$ & $\mathrm{EC}_{90}$ & & $\mathrm{EC}_{50}$ & $\mathrm{EC}_{90}$ \\
\hline AMA & $0.66(2.65 \mu \mathrm{M})$ & $5.93(23.89 \mu \mathrm{M})$ & & $1.79(8.52 \mu \mathrm{M})$ & $16.09(76.58 \mu \mathrm{M})$ \\
AMA-like & $2.16(8.70 \mu \mathrm{M})$ & $19.42(78.26 \mu \mathrm{M})$ & & $0.84(3.99 \mu \mathrm{M})$ & $7.54(35.89 \mu \mathrm{M})$ \\
\hline
\end{tabular}

Data are reported as means and molar concentration is given within parentheses. $\mathrm{EC}_{50}$ and $\mathrm{EC}_{90}=$ 50 and $90 \%$ effective concentrations. 
growth in vitro $\left(\mathrm{EC}_{50}: 0.66 \mu \mathrm{g} / \mathrm{mL}\right.$ or $2.65 \mu \mathrm{M}$ and $\mathrm{EC}_{90}$ : $5.93 \mu \mathrm{g} / \mathrm{mL}$ or $23.9 \mu \mathrm{M})$ than panepoxydone $\left(\mathrm{EC}_{50}: 1.79\right.$ $\mu \mathrm{g} / \mathrm{mL}$ or $8.52 \mu \mathrm{M}$ and $\mathrm{EC}_{90}: 16.09 \mu \mathrm{g} / \mathrm{mL}$ or $\left.76.58 \mu \mathrm{M}\right)$. In contrast, panephoxydone demonstrated a higher inhibitory effect on AMA-like growth than hypnophilin $\left(\mathrm{EC}_{50}\right.$ : $0.84 \mu \mathrm{g} / \mathrm{mL}$ or $3.99 \mu \mathrm{M}$ and $\mathrm{EC}_{90}: 7.54 \mu \mathrm{g} / \mathrm{mL}$ or 35.89 $\mu \mathrm{M})$ than hypnophilin $\left(\mathrm{EC}_{50}: 2.16 \mu \mathrm{g} / \mathrm{mL}\right.$ or $8.70 \mu \mathrm{M}$ and $\mathrm{EC}_{90}: 19.42 \mu \mathrm{g} / \mathrm{mL}$ or $\left.78.26 \mu \mathrm{M}\right)$.

These natural products showed a similar significant inhibitory effect at $1.25 \mu \mathrm{g} / \mathrm{mL}$ (Figure 2B). On the basis of the data in Figure 2A, we suggest that $1.25 \mu \mathrm{g} / \mathrm{mL}(6 \mu \mathrm{M}$ panepoxydone and $5 \mu \mathrm{M}$ hypnophilin) is the concentration guaranteeing higher TR, AMA and AMA-like inhibitory capacity as well as the least inhibition of PBMC proliferation. Interestingly, $5 \mu \mathrm{M}$ hypnophilin is able to block $100 \%$ TR activity and about $70 \%$ of AMA and AMA-like growth, with minor inhibition of PHA-stimulated PBMC proliferation. In contrast, $6 \mu \mathrm{M}$ panepoxydone had a lower $(<10 \%)$ inhibitory effect against TR activity and AMA growth but presented $80 \%$ inhibition of AMA-like growth, but this was also associated with a significant inhibitory effect (50\%) on $\mathrm{PHA}$-induced PBMC proliferation, suggesting that $6 \mu \mathrm{M}$ panepoxydone has cytotoxic activity (Figure 2B).

\section{Hypnophilin displayed a non-cytotoxic immunomodulatory mechanism of action on in vitro human $\mathrm{PBMC}$ cultures}

Figure 3A (PHA-induced proliferation) shows that both $6 \mu \mathrm{M}$ panepoxydone and $5 \mu \mathrm{M}$ hypnophilin inhibited PBMC proliferation, but this effect was more pronounced for panepoxydone. The statistically significant inhibition of proliferation (Figure 3B) was dose-dependent and occurred at a lower concentration of $0.31 \mu \mathrm{g} / \mathrm{mL}(1.3 \mu \mathrm{M})$ for panepoxydone compared to hypnophilin $(2.5 \mu \mathrm{g} / \mathrm{mL}$ or $10.1 \mu \mathrm{M})$.

\section{Incubation of human PBMC with panepoxydone significantly reduced the percentage of CD16 ${ }^{+}$ and CD14 ${ }^{+}$cells and provoked down-regulation of $\mathrm{CD}^{+}{ }^{+}, \mathrm{CD}^{+}$and $\mathrm{CD}^{+}$cells}

We determined the effect of these compounds on lymphocyte subsets or monocytes by immunophenotyping and flow cytometry (Figure 3C). Panepoxydone, $1.25 \mu \mathrm{g} /$ $\mathrm{mL}(6 \mu \mathrm{M})$, significantly reduced the percentage of CD16 ${ }^{+}$ $(1.0 \pm 0.0 \%)$ and CD14 ${ }^{+}$cells $(2.0 \pm 1.0 \%)$ compared to untreated cells (11.0 \pm 4.0 and $11.0 \pm 5.0 \%$, respectively) while hypnophilin did not alter any of the phenotypes analyzed. Moreover, $1.25 \mu \mathrm{g} / \mathrm{mL}(6 \mu \mathrm{M})$ panepoxydone altered the profile of lymphocyte subpopulations by down-regulating $\mathrm{CD} 19^{+}, \mathrm{CD}^{+}$and $\mathrm{CD}^{+}$molecules on the lymphocyte surface. Cytometric data illustrating these findings are presented in Figure 4. Taken together, these results suggest a potential for toxicity by panepoxydone and support the conclusion that hypnophilin had no cytotoxic effect on human PBMC.

\section{Discussion}

We have been investigating the biological activities of hypnophilin and panepoxydone, natural products isolated from $L$. strigosus (12), focusing on identifying new drugs for the treatment of leishmaniasis and Chagas disease. We demonstrate here that hypnophilin is an effective in vitro anti-Trypanosoma and anti-Leishmania compound, with minor toxicity against human PBMC. After appropriate study, this compound could be used as a lead compound for drug design studies against leishmaniasis and Chagas disease.

The $\mathrm{EC}_{50}$ and $\mathrm{EC}_{90}$ values for hypnophilin acting on $\mathrm{AMA}$ were 2.71 and 3.2 times significantly lower, respectively, compared to panepoxydone. In contrast, the $\mathrm{EC}_{50}$ and $\mathrm{EC}_{90}$ values for AMA-like were 2.57 times significantly lower for panepoxydone compared to hypnophilin.

Cytotoxicity assays using normal human PBMC demonstrated that hypnophilin had lower toxicity than panepoxydone. Under our experimental conditions, the results demonstrated that panepoxydone has a typical cytotoxic effect in vitro, suppressing the immune response, leading to a reduction of the percent of lymphocyte and monocyte subpopulations in PBMC cultures, whereas hypnophilin had a non-cytotoxic immunomodulatory mechanism of action.

There is a general consensus that the host immune response plays a pivotal role in controlling adverse events during the massive antigen release triggered by trypanosomicidal agents, by protective responsive that may control tissue damage, diminishing and/or modifying the inflammatory nature of the immune response elicited by the $T$. cruzi antigens released by the action of the drug (18). Hypnophilin at a $5 \mu \mathrm{M}$ concentration has strong anti- $T$. cruzi activity associated with minor non-cytotoxic immunomodulation of human leukocytes and should be effective during the chronic phase of Chagas disease. It is important to note that the currently available treatment for Chagas disease with benznidazole or nifurtimox is effective mainly during the acute phase of infection, with little effect during the chronic phase $(19,20)$. Moreover, the drugs that are available are more effective against the blood flagellates than the intracellular amastigote forms (21). In addition, the drugs currently available to treat the different forms of leishmaniasis were introduced many decades ago and have significant drawbacks, especially in terms of efficacy, length of treatment, route of administration, toxicity, and cost (22). Therefore, we believe that our findings open new perspectives in the field of investigation of the action of natural products of Basidiomycota obtained from Brazilian ecosystems against Chagas disease and Leishmaniasis. Previous studies have identified panepoxydone as an agent interfering with the immune response inhibiting the activation of NF-kappaB $(23,24)$. These findings have led to the proposal that panepoxydone may be useful as an anti-inflammatory agent. In the current study, we investigated, for the first time, 
A

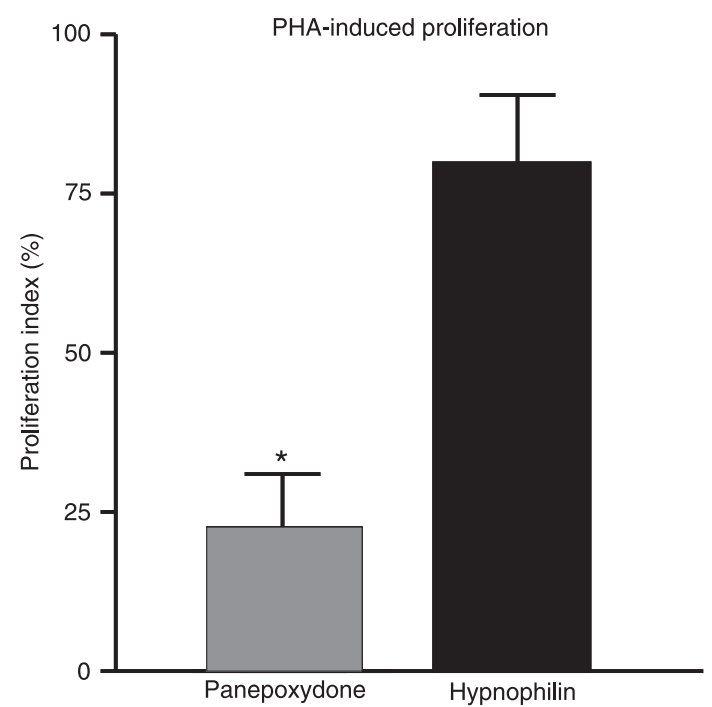

B

Panepoxydone
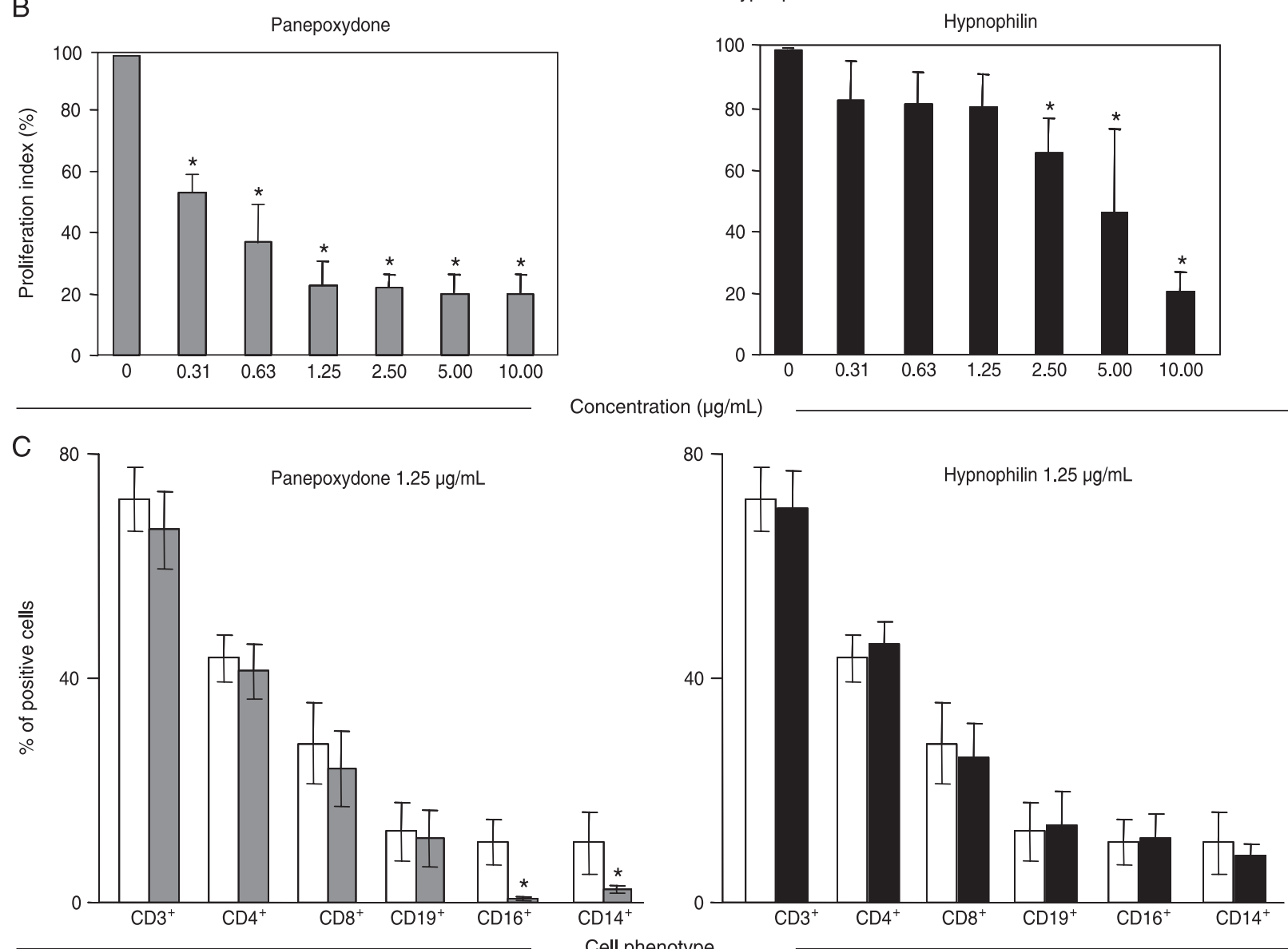

Figure 3. Effect of panepoxydone and hypnophilin on human phytohemagglutinin-induced proliferation and on peripheral blood mononuclear cell (PBMC) subpopulations. A, Human PBMC were incubated with $10 \mu \mathrm{g} / \mathrm{mL}$ panepoxydone (light gray columns) and $10 \mu \mathrm{g} / \mathrm{mL}$ hypnophilin (dark gray columns) for $72 \mathrm{~h}$ and cell proliferation was determined. $B$, Dose-response of proliferation to each compound. $C$, The effect of the substances (filled columns) compared to control (open columns) on lymphocyte subsets (CD $3^{+}, \mathrm{CD}^{+}, \mathrm{CD}^{+}$, CD $19^{+}, \mathrm{CD}_{16}{ }^{+}$cells) and on monocytes (CD14 ${ }^{+}$cells) was determined after $18 \mathrm{~h}$ of incubation followed by flow cytometry analysis. Data are representative of four independent experiments performed in triplicate. ${ }^{*} \mathrm{P}<0.05$ for comparisons between subsets as well as between control cultures (100\% positive; Student $t$-test). 
the effects of panepoxydone on the proliferation of human PBMC stimulated with PHA and on human lymphocyte and monocyte immunophenotyping. Panepoxydone induced a reduction of the percentage of $\mathrm{CD}_{16}{ }^{+}$and $\mathrm{CD} 14^{+}$cells compared to untreated cells. Quantitative flow cytometry experiments in the presence of hypnophilin at $1.25 \mu \mathrm{g} / \mathrm{mL}$ $(5 \mu \mathrm{M})$ showed a reduced proliferation of PBMC stimulated with PHA and no apparent interference with the percentage of human lymphocyte subsets and monocytes (Figure 4). Since the host immune response plays a pivotal role in the adverse events triggered by antigen release during treatment with trypanocidal drugs, the ability of hypnophilin to kill the intracellular forms of T. cruzi or L. (L.) amazonensis, while having no significant cytotoxic effect on human PBMC proliferation, suggests that this terpenoid may be a promising prototype for the development of new chemotherapeutic
A

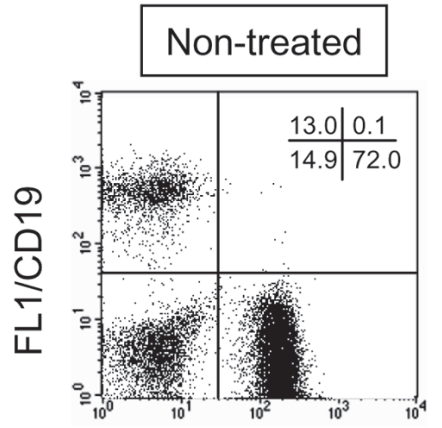

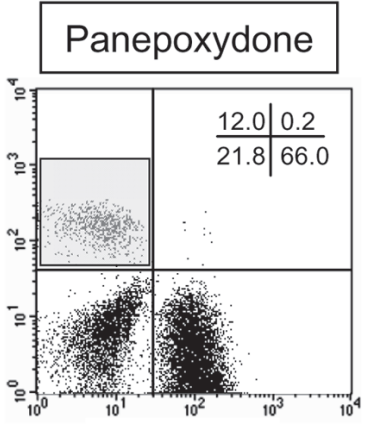

FL2/CD3

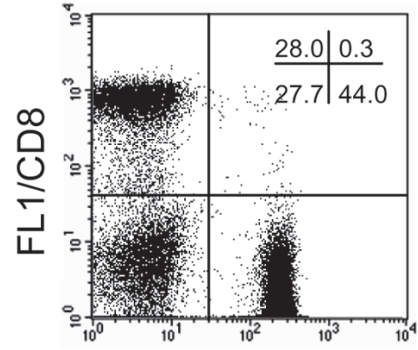

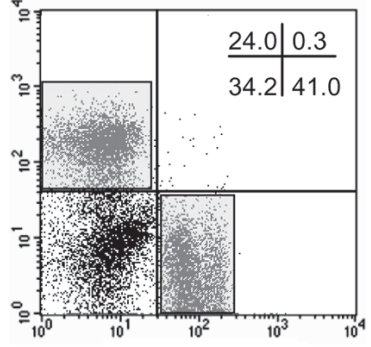

FL2/CD4

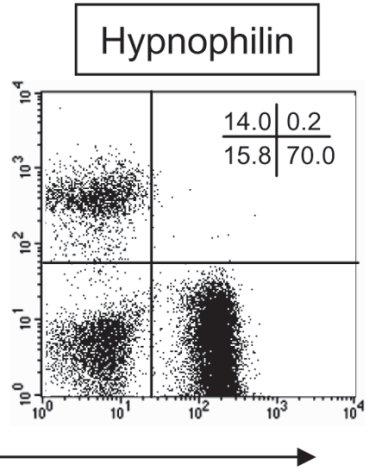

B
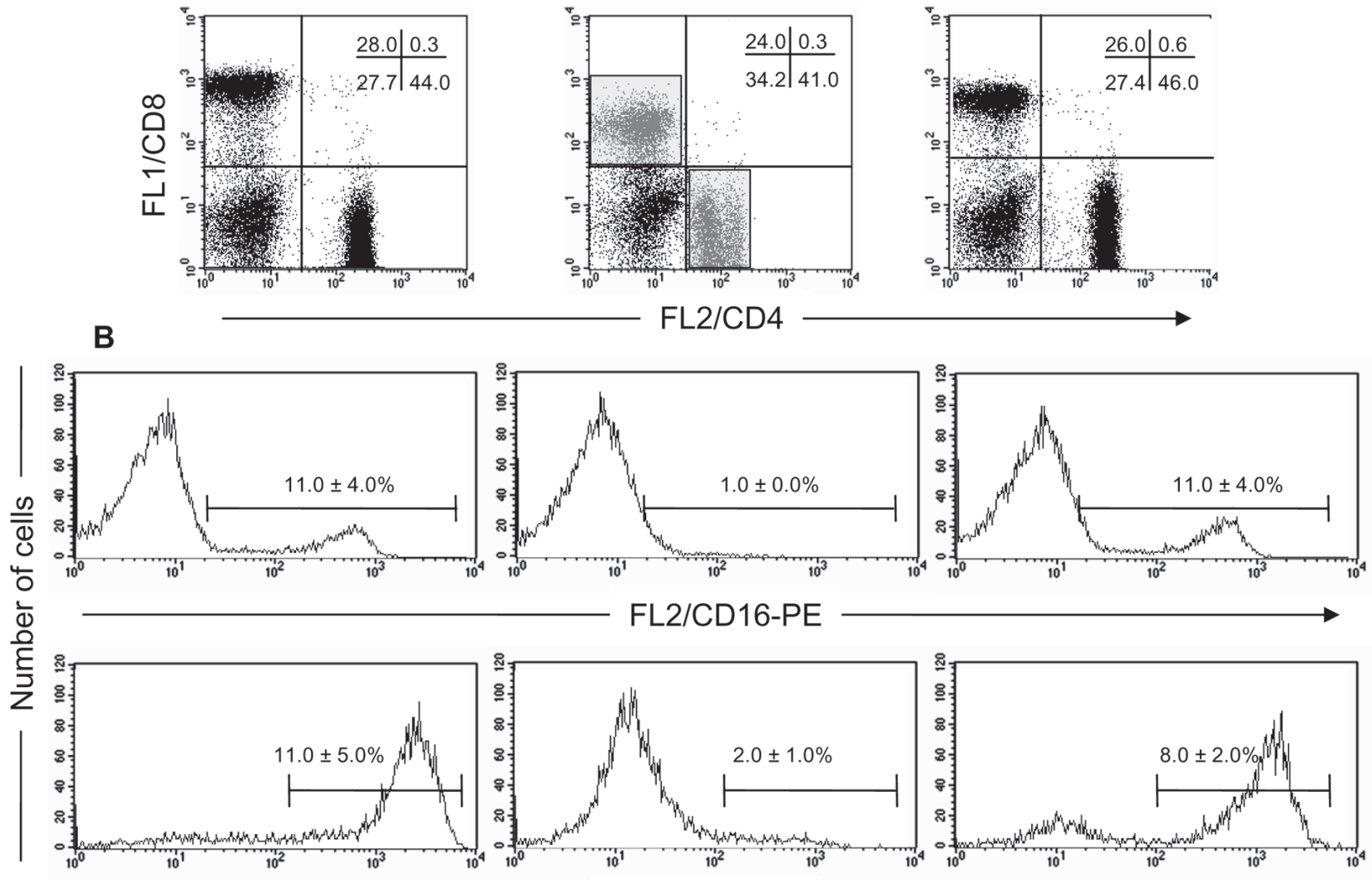

FL2/CD14-PE

Figure 4. Representative data illustrating the effect of panepoxydone and hypnophilin on peripheral blood mononuclear cell subpopulations. Lymphocyte subsets $\left(\mathrm{CD}^{+}, \mathrm{CD}^{+}, \mathrm{CD}^{+}, \mathrm{CD} 19^{+}\right.$cells) are shown in dot plots (A) while $\mathrm{CD}_{16}{ }^{+}$cells and $\mathrm{CD} 14^{+}$cells $/ \mathrm{mono}-$ cytes are shown in histograms (B). The effect of the compounds on both lymphocyte subsets and monocytes was determined after 18 $\mathrm{h}$ of incubation followed by analysis by flow cytometry. 
agents for Chagas disease and leishmaniasis. Additional studies are underway to characterize the molecular basis of hypnophilin-induced immunomodulatory activity.

\section{Acknowledgments}

The authors are grateful to Rosana Alves, Rodrigo Leite and Aline Vaz for technical assistance, to Prof. Alan Fairlamb (University of Dundee, Scotland) for the gift of the recombinant trypanothione reductase, and to Prof. Freder- ick Buckner (University of Washington, USA) for providing the Tulahuen strain of Trypanosoma cruzi expressing the Escherichia coli $\beta$-galactosidase gene. The authors are also grateful to FAPEMIG, CAPES, CNPq, and FIOCRUZ-PDTIS for financial support. The authors also thank the Program for Technological Development in Tools for Health (PDTIS), FIOCRUZ, for the use of its facilities. E.M. Souza-Fagundes, A.J. Romanha, R. Corrêa-Oliveira, C.A. Rosa, C.L. Zani, A. Teixeira-Carvalho, and O.A. Martins-Filho are recipients of CNPq fellowships.

\section{References}

1. Kesselheim AS. Drug development for neglected diseases the trouble with FDA review vouchers. N Engl J Med 2008; 359: 1981-1983.

2. Vermeersch M, Foubert K, da Luz RI, Van Puyvelde L, Pieters L, Cos P, et al. Selective anti-Leishmania activity of 13,28-epoxy-oleanane and related triterpene saponins from the plant families Myrsinaceae, Primulaceae, Aceraceae and Icacinaceae. Phytother Res 2009; 23: 1404-1410.

3. Boiani L, Aguirre G, Gonzalez M, Cerecetto H, Chidichimo A, Cazzulo JJ, et al. Furoxan-, alkylnitrate-derivatives and related compounds as anti-trypanosomatid agents: mechanism of action studies. Bioorg Med Chem 2008; 16: 79007907.

4. Coura JR. Present situation and new strategies for Chagas disease chemotherapy - a proposal. Mem Inst Oswaldo Cruz 2009; 104 (Suppl 4): 549-554.

5. Cançado JR. Long term evaluation of etiological treatment of Chagas disease with benznidazole. Rev Inst Med Trop São Paulo 2002; 44: 29-37.

6. Rodriques Coura J, de Castro SL. A critical review on Chagas disease chemotherapy. Mem Inst Oswaldo Cruz 2002; 97: 3-24.

7. Cançado JR. Tratamento específico. In: Cançado JR, Chuster M (Editors), Cardiopatia chagásica. Rio de Janeiro: Fundação Carlos Chagas; 1985.

8. Krauth-Siegel RL, Inhoff O. Parasite-specific trypanothione reductase as a drug target molecule. Parasitol Res 2003; 90 (Suppl 2): S77-S85

9. Krauth-Siegel RL, Shöneck R. Trypanothione reductase and lipoamide dehydrogenase as targets for a structure-based drug design. FASEBJ 1995; 9: 1138-1146.

10. Souza-Fagundes EM, Gazzinelli G, Parreira GG, MartinsFilho OA, Amarante-Mendes GP, Correa-Oliveira R, et al. In vitro activity of labdane diterpene from Alomia myriadenia (Asteraceae): immunosuppression via induction of apoptosis in monocytes. Int Immunopharmacol 2003; 3: 383-392.

11. Pegler DN. The genus Lentinus: a world monograph. London: Her Majesty's Stationery Office, Kew Bulletin Additional Series 10; 1983.

12. Cota BB, Rosa LH, Fagundes EM, Martins-Filho OA, Correa-Oliveira R, Romanha AJ, et al. A potent trypanocidal component from the fungus Lentinus strigosus inhibits trypanothione reductase and modulates PBMC proliferation. Mem Inst Oswaldo Cruz 2008; 103: 263-270.

13. Hamilton CJ, Saravanamuthu A, Eggleston IM, Fairlamb AH. Ellman's-reagent-mediated regeneration of trypanothione in situ: substrate-economical microplate and time-dependent inhibition assays for trypanothione reductase. Biochem $J$ 2003; 369: 529-537.

14. Borges A, Cunningham ML, Tovar J, Fairlamb AH. Sitedirected mutagenesis of the redox-active cysteines of Trypanosoma cruzi trypanothione reductase. Eur J Biochem 1995; 228: 745-752.

15. Buckner FS, Verlinde CL, La Flamme AC, Van Voorhis WC. Efficient technique for screening drugs for activity against Trypanosoma cruzi using parasites expressing beta-galactosidase. Antimicrob Agents Chemother 1996; 40: 25922597.

16. Jiang $\mathrm{J}, \mathrm{Xu} \mathrm{Q}$. Immunomodulatory activity of the aqueous extract from rhizome of Smilax glabra in the later phase of adjuvant-induced arthritis in rats. J Ethnopharmacol 2003; 85: 53-59.

17. Gazzinelli G, Katz N, Rocha RS, Colley DG. Immune responses during human schistosomiasis mansoni. X. Production and standardization of an antigen-induced mitogenic activity by peripheral blood mononuclear cells from treated, but not active cases of schistosomiasis. J Immunol 1983; 130: 2891-2895.

18. Sathler-Avelar R, Vitelli-Avelar DM, Massara RL, Borges JD, Lana M, Teixeira-Carvalho A, et al. Benznidazole treatment during early-indeterminate Chagas' disease shifted the cytokine expression by innate and adaptive immunity cells toward a type 1-modulated immune profile. Scand J Immunol 2006; 64: 554-563.

19. Tanowitz HB, Kirchhoff LV, Simon D, Morris SA, Weiss LM, Wittner M. Chagas' disease. Clin Microbiol Rev 1992; 5: 400-419.

20. Prata A. Clinical and epidemiological aspects of Chagas disease. Lancet Infect Dis 2001; 1: 92-100.

21. Muelas-Serrano S, Le-Senne A, Fernandez-Portillo C, Nogal JJ, Ochoa C, Gomez-Barrio A. In vitro and in vivo anti-Trypanosoma cruzi activity of a novel nitro-derivative. Mem Inst Oswaldo Cruz 2002; 97: 553-557.

22. Den Boer ML, Alvar J, Davidson RN, Ritmeijer K, Balasegaram M. Developments in the treatment of visceral leishmaniasis. Exp Op Emerg Drugs 2009; 14: 395-410.

23. Umezawa K, Ariga A, Matsumoto N. Naturally occurring and synthetic inhibitors of NF-kappaB functions. Anticancer Drug Des 2000; 15: 239-244.

24. Erkel G, Wisser G, Anke T. Influence of the fungal NFkappaB inhibitor panepoxydone on inflammatory gene expression in MonoMac6 cells. Int Immunopharmacol 2007; 7: 612-624. 